\title{
SOME REMARKS ON LEVI COMPLEMENTS AND ROOTS IN LIE ALGEBRAS WITH CONE POTENTIAL
}

\author{
by KARLHEINZ SPINDLER
}

(Received 26th February 1990, revised 9th September 1990)

\begin{abstract}
We study Lie algebras with cone potential which play a prominent role in the Lie theory of semigroups. For these algebras, we obtain a uniqueness theorem for Levi complements and information on the fine structure of the root system.
\end{abstract}

1980 Mathematics subject classification (1985 Revision): 22E60, $17 \mathrm{~B} 05$.

\section{Introduction}

An important part of the Lie theory of semigroups is the study of invariant cones in Lie algebras. It turns out that the existence of an invariant pointed cone with inner points in a real Lie algebra $L$ imposes severe restrictions on the structure of $L$. First of all, $L$ possesses compactly embedded Cartan algebras. As all of these are conjugate, it's no harm to fix one such, say $H$. Now $L$ as an $H$-module decomposes into the zero-module $L^{0}=H$ and a complementary module $L^{+}$which in turn can be split up into its isotypic components. In order to parameterize these, one uses a certain set $\Omega$ of linear forms on $H$ which are called the roots of the pair $(L, H)$. These linear forms arise in pairs $(\omega,-\omega)$. Choosing one element from each pair, one gets a set $\Omega^{+}$and a complex structure $I: L^{+} \rightarrow L^{+}$. Now the above-mentioned decomposition of $L$ into its isotypic components can be written down as

$$
L=H \oplus \bigoplus_{\omega \in \mathbf{\Omega}^{+}} L^{\omega},
$$

where

$$
L^{\omega}=\{x \in L \mid[h, x]=\omega(h) I x \text { for all } h \in H\}
$$

This is an orthogonal decomposition with respect to the Cartan-Killing form $B$ of $L$. 
Furthermore, there is a unique maximal compactly embedded subalgebra of $L$ containing $H$. We denote this algebra by $K_{H}$, its center by $Z\left(K_{H}\right)$.

Let comp $L$ denote the set of compact elements of $L$, i.e., those elements $x \in L$ with $e^{\overline{R a d} x}$ compact. The Lie algebra $L$ is called quasi-hermitian if $Z\left(K_{H}\right) \cap(\operatorname{comp} L)^{0} \neq \emptyset$. If $L$ is quasi-hermitian, the set of roots decomposes into two disjoint classes, namely the class $\Omega_{K}$ of those roots $\omega$ with $L^{\omega} \subseteq K_{H}$ and the class $\Omega_{P}$ of those roots $\omega$ with $L^{\omega} \cap K_{H}=\{0\}$. The two types are called compact roots and non-compact roots, respectively. Writing

$$
P_{H}:=\bigoplus_{\omega \in \Omega_{\mathbf{P} \cap \Omega^{+}}} L^{\omega},
$$

we have the decomposition $L=K_{H} \oplus P_{H}$ where $\left[K_{H}, P_{H}\right] \subseteq P_{H}$.

Finally, $L$ is said to have cone potential if $[I x, x] \neq 0$ whenever $x \neq 0$ comes from one of the root spaces $L^{\omega}$. Let us denote by $Z, N$ and $R$ the centre, the nilradical and the radical of $L$, respectively. Now if $L$ has cone potential, then $N \cap L^{\omega}=R \cap L^{\omega}$ for all $\omega \in \Omega$, and $[N, N] \subseteq Z$. Previously, the author has obtained a universal construction for Lie algebras with cone potential (see [6, (4.3), (4.4), pp. 76-82]) saying the following: Any Lie algebra with cone potential is a direct sum of an abelian algebra and a quotient of an algebra.

$$
M \oplus\left(\bigwedge^{2} M\right)_{0} \oplus \mathfrak{g}
$$

where $\mathrm{g}$ is a reductive algebra and where $M$ is a $\mathrm{g}$-module such that a Cartan algebra $\mathfrak{h}$ of $g$ acts compactly and effectively on $M$ in a certain way such that $\left(\bigwedge^{2} M\right)_{0} \neq\{0\}$. For the rest of this paper, we shall fix a real Lie algebra $L$ with a compactly embedded Cartan algebra $H$. As above, let us denote the centre, the nilradical and the radical of $L$ by $Z, N$ and $R$, respectively.

Some constructions which are important in the theory of invariant cones depend on the fact that a suitable Levi complement $S$ can be chosen. In this paper we shall show the uniqueness of such a suitable $S$ (once $H$ is fixed) and draw some conclusions.

The leading theme in our investigations is the question to what extent the structure of a Lie algebra is determined by the existence of an invariant cone. Theorems 2 and 3 below give some further results in this direction. One can hope that eventually an effective classification of Lie algebras with invariant cones can be given.

Definition. Let $L$ be a Lie algebra with radical $R$. A Cartan algebra $H$ and a Levi complement $S$ will be called adapted if the decomposition

$$
H=(H \cap R) \oplus(H \cap S)
$$

holds. The following theorem is well-known (see [2, VII.3, Ex. 11, pp. 61-62)] or [6, (2.7), pp. 44-45]).

Theorem. Let $L$ be a Lie algebra over a field of characteristic 0 with radical $R$. 
(a) Given a Levi complement $S$ and a Cartan algebra $H_{0}$ of $S$, there is a subalgebra $U \leqq R$ such that $H:=H_{0}+U$ is a Cartan algebra of $L$. (Note that $H_{0}=H \cap S$ and $U=H \cap R$ in this case, so that $H$ and $S$ are adapted.)

(b) Given a Cartan algebra $H$ of $L$, there exist a Levi complement $S$, a Cartan algebra $H_{0}$ of $S$ and a subalgebra $U \leqq R$ such that $H=H_{0}+U$. (Note that $H_{0}=H \cap S$ and $U=H \cap R$ in this case, so that $S$ and $H$ are adapted.)

In particular, to any Cartan algebra $H$ we can find a Levi complement adapted to $H$. In the theory of invariant cones, it turns out that one has to use a suitable Levi complement with additional properties. For the proof of the following theorem, we refer to $[4$, III. 6.28, pp. $227-228]$ or $6,(3.7)$, pp. 67-68].

Theorem. If $L$ is a real Lie algebra with a compactly embedded Cartan algebra $H$, there is a Levi complement $S$ adapted to $H$ such that

$$
[H \cap R, S]=\{0\}
$$

For any such Levi complement, the following assertions hold.

(1) $H \cap S$ is a compactly embedded Cartan algebra of $S$.

(2) $H+S=(H \cap R) \oplus S$, and this is a reductive Lie algebra.

(3) $[H, S] \subseteq S$.

(4) $L^{\omega}=\left(L^{\omega} \cap R\right) \oplus\left(L^{\omega} \cap S\right)$ for all $\omega \in \Omega$.

(5) $R=(H \cap R) \oplus\left(L^{+} \cap R\right)$ is a direct sum of $(H+S)$-modules.

In this paper, we shall prove that in a Lie algebra with cone potential such a Levi complement is uniquely determined. The method of proof will give further information on the structure of the root system.

\section{Lie algebra modules $V$ with $\left(\bigwedge^{2} V\right)_{0} \neq\{0\}$}

A representation $\rho: L \rightarrow$ End $V$ induces a representation $\wedge^{p} \rho: L \rightarrow$ End $\wedge^{p} V$ given by

$$
\left(\wedge^{p} \rho\right)(x)(v \wedge w)=(\rho(x) v) \wedge w+v \wedge(\rho(x) w)
$$

For later purposes, we want to investigate the zero-module $\left(\bigwedge^{p} V\right)_{0}$ of this induced representation. Although we will be only interested in the case $p=2$, we treat the general case because no extra effort is needed. With the canonical pairing

$$
\widetilde{\Omega}(\xi)[\Phi]:=\Phi(\xi) \quad\left(\Phi \in\left(\bigwedge^{p} V\right)^{*}, \xi \in \bigwedge^{p} V\right)
$$

the diagram 


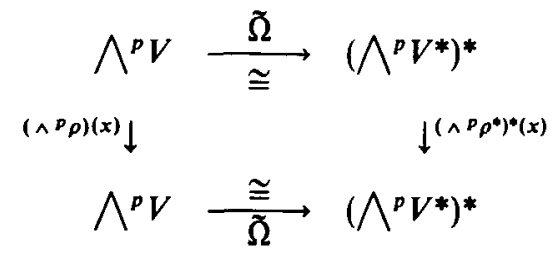

commutes so that $\Omega$ intertwines the representations $\wedge^{p} \rho$ and $\left(\wedge^{p} \rho^{*}\right)^{*}$, i.e., is an isomorphism of modules. (Here and in the sequel, we identify $\left(\wedge^{p} V\right)^{*}$ and $\wedge^{p} V^{*}$ as modules.)

By the universal property of $\bigwedge^{p} V^{*}$ we can identify the alternating $p$-forms on $V^{*}$ with the linear forms on $\bigwedge^{p} V^{*}$ :

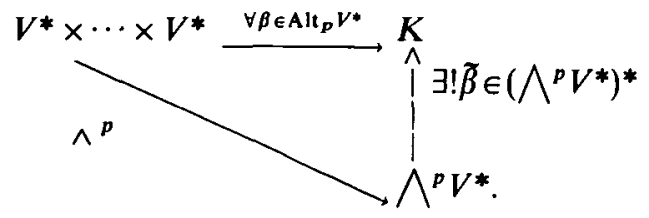

Now a $p$-form $\beta$ on $V^{*}$ is called $\rho^{*}$-invariant, if $\sum_{k=1}^{p} \beta\left(\phi_{1}, \ldots, \rho^{*}(x) \phi_{k}, \ldots, \phi_{p}\right)=0$ for all $\phi_{i} \in V^{*}$ and all $x \in L$. If $\beta$ is alternating, this is the case if and only if

$$
\begin{aligned}
0 & =\sum_{k=1}^{p} \beta\left(\phi_{1}, \ldots, \rho^{*}(x) \phi_{k}, \ldots, \phi_{p}\right) \\
& =\sum_{k=1}^{p} \widetilde{\beta}\left(\phi_{1} \wedge \cdots \wedge \rho^{*}(x) \phi_{k} \wedge \cdots \wedge \phi_{p}\right)=\widetilde{\beta}\left(\left(\wedge^{p} \rho^{*}\right)(x)\left(\phi_{1} \wedge \cdots \wedge \phi_{p}\right)\right)
\end{aligned}
$$

for all $\phi_{i} \in V^{*}$ and all $x \in L$, i.e., if and only if $\not{\beta}$ annihilates $\left(\wedge^{p} \rho^{*}\right)(L)\left(\bigwedge^{p} V^{*}\right)$. Now $\tilde{\Omega}: \bigwedge^{p} V \rightarrow\left(\bigwedge^{p} V^{*}\right)^{*}$ is associated with the isomorphism $\Omega: \bigwedge^{p} V \rightarrow$ Alt $_{p} V^{*}$ which is given by $\Omega(\xi)\left(\phi_{1}, \ldots, \phi_{p}\right)=\Omega(\xi)\left(\phi_{1} \wedge \cdots \wedge \phi_{p}\right)$. We see that an element $\xi \in \wedge^{p} V$ belongs to the zero-module $\left(\bigwedge^{p} V\right)_{0}$ if and only if $\left(\wedge^{p} \rho\right)(x)(\xi)=0$ for all $x \in L$; i.e., if and only if

$$
0=\Phi\left(\left(\wedge^{p}\right)(x) \xi\right)=-\left(\left(\wedge^{p} \rho\right)^{*}(x) \Phi\right)[\xi]=-\Omega(\xi)\left(\left(\wedge^{p} \rho\right)^{*}(x) \Phi\right)
$$

for all $x \in L$ and all $\Phi \in\left(\bigwedge^{p} V\right)^{*}$. Clearly, this condition holds if and only if $\Omega(\xi)$ annihilates $\left(\wedge^{p} \rho^{*}\right)(L)\left(\wedge^{p} V^{*}\right)$, i.e., if and only if $\Omega(\xi)$ is invariant. Hence we have

$$
\Omega\left(\left(\bigwedge^{p} V\right)_{0}\right)=\mathrm{Alt}_{p}^{\mathrm{inv}} V^{*}
$$

where Alt inv $V^{*}$ denotes the space of invariant alternating $p$-forms on $V^{*}$. In particular, $\Omega\left(\bigwedge^{2} V\right)_{0}=\operatorname{Alt}_{2}^{\text {inv }} V^{*}$ is just the space of invariant skew-symmetric bilinear forms on $V^{*}$. 
Now recall that a symplectic form on a vector space is a non-degenerate, skewsymmetric bilinear form. We shall prove the following result.

Proposition 1. If $V$ is any Lie algebra module, then $\left(\bigwedge^{2} V\right)_{0} \cong \mathrm{Alt}_{2}^{\text {inv }} V$. If $V$ is irreducible, then $\left(\bigwedge^{2} V\right)_{0} \neq\{0\}$ if and only if $V$ possesses an invariant symplectic form. In particular, $\operatorname{dim} V$ must be even in this case.

Proof. By the discussion above we have $\left(\bigwedge^{2} V\right)_{0} \cong \mathrm{Alt}_{2}^{\mathrm{inv}} V^{*}$. Now let us observe that Alt ${ }_{2}^{\text {inv }} V^{*} \cong \mathrm{Alt}{ }_{2}^{\text {inv }} V$. To see this, choose a basis $B=\left(v_{1}, \ldots, v_{n}\right)$ of $V$ and take the corresponding dual basis $B^{*}=\left(\phi_{1}, \ldots, \phi_{n}\right)$ on $V^{*}$ which is given by $\phi_{i}\left(v_{j}\right)=\delta_{i j}$. Fix an arbitrary element $x \in L$. If $\rho(x) e_{j}=\sum_{i=1}^{n} x_{i j} e_{i}$ for all $j$, then $\rho^{*}(x) \phi_{j}=-\sum_{i=1}^{n} x_{j i} \phi_{i}$ because we have

$$
\begin{aligned}
\left(\rho^{*}(x) \phi_{j}\right)\left(e_{k}\right) & =-\phi_{j}\left(\rho(x) e_{k}\right)=-\phi_{j}\left(\sum_{i=1}^{n} x_{i k} e_{i}\right)=-\sum_{i=1}^{n} x_{i k} \delta_{j i}=-x_{j k} \\
& =-\sum_{i=1}^{n} x_{j i} \delta_{i k}=\left(-\sum_{i=1}^{n} x_{j i} \phi_{i}\right)\left(e_{k}\right) \quad \text { for all } k .
\end{aligned}
$$

So if we denote the matrix representation of a linear operator $\theta$ with respect to a basis $B$ by $\theta_{B}$ we have shown that $\rho^{*}(x)_{B^{*}}=-\left(\rho(x)_{B}\right)^{T}$ for all $x \in L$.

To any bilinear form $\alpha: V \times V \rightarrow \mathbb{R}$ there is associated a unique matrix $A$ satisfying $\alpha\left(\sum_{i} x_{i} v_{i}, \sum_{j} y_{j} v_{j}\right)=x^{T} A y$. Saying that $\alpha$ is non-degenerate, skew-symmetric, and $\rho$ invariant is tantamount saying

$$
\operatorname{det} A \neq 0, \quad A^{T}=-A, \quad X^{T} A+A X=0 \text { for all } X=\rho(x)_{B}(x \in L) .
$$

Analogously, a bilinear form $\beta$ on $V^{*}$ is in $\operatorname{Alt}_{2}^{\text {inv }} V^{*}$ if and only if its matrix $B$ with respect to the basis $B^{*}$ satisfies

$$
\operatorname{det} B \neq 0, \quad B^{T}=-B, \quad X B+B X^{T}=0 \text { for all } X=\rho(x)_{B}(x \in L)
$$

here we used the fact that $\rho^{*}(x)_{B^{*}}=-\left(\rho(x)_{B}\right)^{T}$. Now it is easy to check that putting $B:=A^{-1}$ yields an isomorphism between Alt ${ }_{2}^{\text {inv }}$ and $\mathrm{Alt}_{2}^{\mathrm{inv}} V^{*}$.

If the module $V$ is irreducible, an element $\alpha \in \mathrm{Alt}_{2}^{\mathrm{inv} V}$ is either non-degenerate or the zero-mapping. To see this, observe that the radical

$$
\operatorname{Rad} \alpha:=\{v \in V \mid \alpha(v, w)=0 \text { for all } w \in V\}
$$

is a submodule of $V$, due to the skew-symmetry of $\alpha$. As $V$ is irreducible, this is possible only if $\operatorname{Rad} \alpha=V$ (which means $\alpha=0$ ) or $\operatorname{Rad} \alpha=\{0\}$ (which means the non-degeneracy of $\alpha$ ). Thus the non-zero elements of $\mathrm{Alt}_{2}^{\mathrm{inv}} V$ are precisely the invariant symplectic forms. This gives the result. 
Before we can prove our next result we provide some background for the representation theory of $\operatorname{so}(3)$ and $\operatorname{sl}(2, \mathbb{R})$.

Let $V_{n}$ be the space of all homogeneous complex polynomials of degree $n$ in two variables. Then $S U(2)$ acts canonically on $V_{n}$. It is well-known $([1, \mathrm{II} .5$, pp. 84-86; also 7]) that the spaces $V_{n}\left(n \in \mathbb{N}_{0}\right)$ form a complete collection of (equivalence classes of) irreducible representations of $S U(2)$. Since $s o(3) \cong s u(2)$ and since $S U(2)$ is simply connected, the spaces $V_{n}$ are exactly the irreducible complex so(3)-modules. From the representation theory of $S U(2)$, we can thus easily deduce the following fact which we will need later on: Let $\left(e_{1}, e_{2}, e_{3}\right)$ be a basis of $\operatorname{so}(3)$ with $\left[e_{i}, e_{i+1}\right]=e_{i+2}$. If an irreducible complex representation $\rho$ of so(3) is such that $\rho\left(e_{1}\right)$ is invertible, then no weight of $\rho$ takes any of the values $\pm 2 i$ on $e_{1}$.

From the well-known classification of the irreducible sl $(2, \mathbb{C})$-modules $([5$, IV, 2-4]) one easily deduces that of $\operatorname{sl}(2, \mathbb{R})$; complete calculations can be found in $[3,11.2, \mathrm{pp}$. 3948]. The result is as follows. For any $\lambda \in \mathbb{N}$, there is (up to isomorphism) a unique irreducible $\mathbf{s l}(2, \mathbb{R})$-module $V^{\lambda}$ with $\operatorname{dim} V^{\lambda}=\lambda+1$. To describe this module, we put

$$
N:= \begin{cases}\frac{\lambda}{2}-1, & \text { if } \lambda \text { is even } \\ \frac{\lambda-1}{2}, & \text { if } \lambda \text { is odd }\end{cases}
$$

in the sequel all terms with $N+1$ have to be ignored in the case that $\lambda$ is odd. Now we can choose a basis $\left(F_{0}, I F_{0}, F_{1}, I F_{1}, \ldots, F_{N}, I F_{N}, F_{N+1}\right)$ of $V^{\lambda}$ such that the elements

$$
U:=\left(\begin{array}{rr}
0 & 1 \\
-1 & 0
\end{array}\right), \quad T:=\left(\begin{array}{ll}
0 & 1 \\
1 & 0
\end{array}\right), \quad H:=\left(\begin{array}{rr}
1 & 0 \\
0 & -1
\end{array}\right)
$$

with the bracket relations

$$
[U, T]=2 H, \quad[U, H]=-2 T, \quad[T, H]=-2 U
$$

satisfy the following conditions for $0 \leqq n \leqq N$ :

$$
\begin{array}{ll}
U * F_{n}=(\lambda-2 n) I F_{n}, & U * I F_{n}=-(\lambda-2 n) F_{n}, \\
T * F_{n}=(\lambda-n) I F_{n+1}-n I F_{n-1}, & T * I F_{n}=-(\lambda-n) F_{n+1}+n F_{n-1}, \\
H * F_{n}=(\lambda-n) F_{n+1}+n F_{n-1}, & H * I F_{n}=(\lambda-n) I F_{n+1}+n I F_{n-1} .
\end{array}
$$

If $\lambda$ is even, then additionally

$$
U * F_{N+1}=T * F_{N+1}=H * F_{N+1}=0
$$

In other words: With respect to this basis, $U, T$ and $H$, considered as operators on $V^{\lambda}$, are given as matrices 


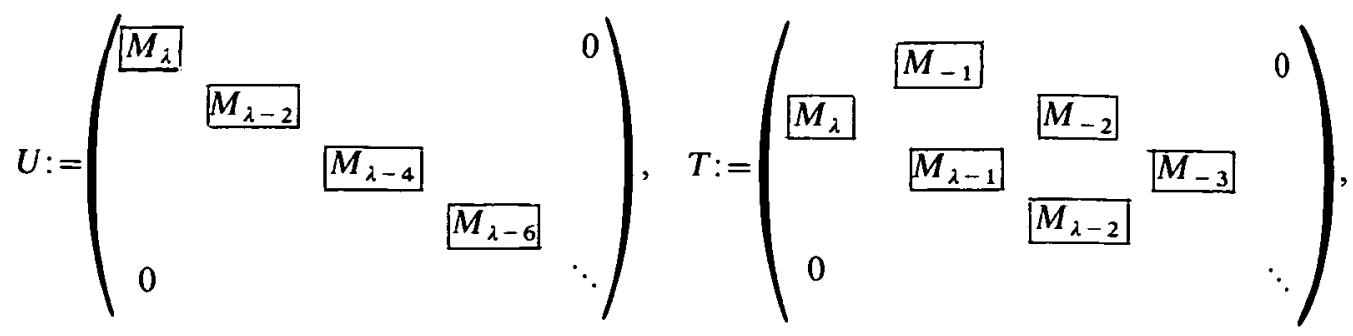

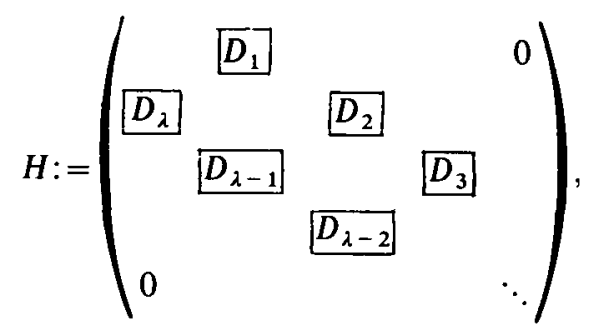

where

$$
M_{\alpha}:=\left(\begin{array}{rr}
0 & -\alpha \\
\alpha & 0
\end{array}\right) \text { and } D_{\alpha}:=\left(\begin{array}{ll}
\alpha & 0 \\
0 & \alpha
\end{array}\right)
$$

If $\lambda$ is even, in all matrices a zero row and a zero column must be added.

Proposition 2. Suppose that $V^{\lambda}$ is the irreducible $\operatorname{sl}(2, \mathbb{R})$-module of dimension $\lambda+1$ with the basis given above. Then the zero submodule $\left(\bigwedge^{2} V^{\lambda}\right)_{0}$ of $\bigwedge^{2} V^{\lambda}$ is $\{0\}$ if $\lambda$ is even and

$$
\mathbb{R}\left(\sum_{n=0}^{N}(-1)^{n}\left(\begin{array}{l}
\lambda \\
n
\end{array}\right)\left(F_{n} \wedge I F_{n}\right)\right)
$$

if $\lambda=2 N+1$ is odd.

Proof. We want to compute $\left(\bigwedge^{2} V^{\lambda}\right)_{0}$. If $\lambda$ is even, then $\operatorname{dim} V^{\lambda}$ is odd, whence $\left(\wedge^{2} V^{\lambda}\right)_{0}=\{0\}$. So suppose $\lambda=2 N+1$ is odd. A basis of $\wedge^{2} V^{\lambda}$ is given by the vectors $F_{m} \wedge F_{n}$ and $I F_{m} \wedge I F_{n}$ where $0 \leqq m<n \leqq N$ and the vectors $F_{m} \wedge I F_{n}$ where $0 \leqq m, n \leqq N$ are arbitrary. Now remember that the action of $X \in \mathrm{sl}(2, \mathbb{R})$ on $\wedge^{2} V^{\lambda}$ is given by $X *(v \wedge w)=(X v) \wedge w+v \wedge(X w)$. An element

$$
\xi=\sum_{m<n} \alpha_{m n}\left(F_{m} \wedge F_{n}\right)+\sum_{m<n} \beta_{m n}\left(I F_{m} \wedge I F_{n}\right)+\sum_{m, n} \gamma_{m n}\left(F_{m} \wedge I F_{n}\right)
$$

vanishes under the action of $U$ if and only if 


$$
\begin{aligned}
0= & U \xi=\sum_{m<n} \alpha_{m n}\left((\lambda-2 m)\left(I F_{m} \wedge F_{n}\right)+(\lambda-2 n)\left(F_{m} \wedge I F_{n}\right)\right) \\
& +\sum_{m<n} \beta_{m n}\left(-(\lambda-2 m)\left(F_{m} \wedge I F_{n}\right)-(\lambda-2 n)\left(I F_{m} \wedge F_{n}\right)\right) \\
& +\sum_{m, n} \gamma_{m n}\left((\lambda-2 m)\left(I F_{m} \wedge I F_{n}\right)-(\lambda-2 n)\left(F_{m} \wedge F_{n}\right)\right. \\
= & \sum_{m<n}\left(\alpha_{m n}(\lambda-2 m)-\beta_{m n}(\lambda-2 n)\right)\left(I F_{m} \wedge F_{n}\right) \\
& +\sum_{m<n}\left(\alpha_{m n}(\lambda-2 n)-\beta_{m n}^{\beta_{m}(\lambda-2 m)}\right)\left(F_{m} \wedge F_{n}^{\left.I F_{n}\right)}\right. \\
& +\sum_{m, n} \gamma_{m n}((\lambda-2 m)(\underbrace{I F_{m} \wedge I F_{n}}_{=0 \text { for } m=n})-(\lambda-2 n) \underbrace{\left(F_{m} \wedge F_{n}\right)}_{=0 \text { for } m=n})
\end{aligned}
$$

i.e., $U * \xi=0$ if and only if $\gamma_{m n}=0$ for all $m \neq n$ and

$$
\left(\begin{array}{ll}
\lambda-2 m & -(\lambda-2 n) \\
\lambda-2 n & -(\lambda-2 m)
\end{array}\right)\left(\begin{array}{l}
\alpha_{m n} \\
\beta_{m n}
\end{array}\right)=\left(\begin{array}{l}
0 \\
0
\end{array}\right)
$$

or equivalently $\alpha_{m n}=\beta_{m n}=0$ for all $m<n$. (Note that

$$
\operatorname{det}\left(\begin{array}{ll}
\lambda-2 m & -(\lambda-2 n) \\
\lambda-2 n & -(\lambda-2 m)
\end{array}\right)=-(\lambda-2 m)^{2}+(\lambda-2 n)^{2}
$$

does not vanish.) There is no restriction for the coefficients $\gamma_{n n}(0 \leqq n \leqq N)$. So we have proved that the null space of $U$ in $\wedge^{2} V^{\lambda}$ is

$$
\sum_{n=0}^{N} \mathbb{R}\left(F_{n}^{\prime} \wedge I F_{n}\right)
$$

We could have seen this also in a non-computational way. Putting $V_{n}^{\lambda}:=\mathbb{R} F_{n} \oplus \mathbb{R} I F_{n}$, the decomposition of $V^{\lambda}$ into the irreducible $\mathbb{R} U$-modules is given by

$$
V^{\lambda}=\bigoplus_{n} V_{n}^{\lambda}
$$

Using the isomorphism

$$
\bigwedge^{2} V^{\lambda} \cong\left(\bigoplus_{n}\left(\bigwedge^{2} V_{n}^{\lambda}\right)\right) \oplus\left(\bigoplus_{i \neq j}\left(V_{i}^{\lambda} \otimes V_{j}^{\lambda}\right)\right)
$$


of $\mathbb{R} U$-modules, we obtain

$$
\left(\bigwedge^{2} V^{\lambda}\right)_{0} \cong\left(\bigoplus _ { n } ^ { \oplus } ( \underbrace { \bigwedge ^ { 2 } V _ { n } ^ { \lambda } ) _ { 0 } } _ { = \bigwedge ^ { 2 } V _ { n } ^ { \lambda } } ) \oplus \left(\bigoplus_{i \neq j}^{\oplus}(\underbrace{\left.V_{i}^{\lambda} \otimes V_{j}^{\lambda}\right)_{0}}_{=\{0\}}\}\right.\right.
$$

where the subscript ' 0 ' denotes the zero modules of the action of $U$ alone.

To see that $\left(\bigwedge^{2} V_{n}^{\lambda}\right)_{0}=\bigwedge^{2} V_{n}^{\lambda}=\mathbb{R}\left(F_{n} \wedge I F_{n}\right)$, we observe that

$$
U *\left(F_{n} \wedge I F_{n}\right)=\left(U F_{n} \wedge I F_{n}\right)+\left(F_{n} \wedge U I F_{n}\right)=((\lambda-2 n)-(\lambda-2 n))\left(F_{n} \wedge I F_{n}\right)=0
$$

more generally, every one-dimensional $\mathbb{R} U$-module is a zero module if it is obtained by restriction of an $\operatorname{sl}(2, \mathbb{R})$-action since $e^{\mathbf{R U}}$ is compact.

To see that $\left(V_{i}^{\lambda} \otimes V_{j}^{\lambda}\right)_{0}=\{0\}$ we just state that $\operatorname{Hom}_{U}\left(V_{i}^{\lambda}, V_{j}^{\lambda}\right)=\{0\}$ for $i \neq j$.

Now we ask when an element $\xi=\sum_{n=0}^{N} c_{n}\left(F_{n} \wedge I F_{n}\right)$ in the null space of $U$ is also annihilated by $T$. From

$$
T\left(F_{n} \wedge I F_{n}\right)=(\lambda-n)\left(I F_{n+1} \wedge I F_{n}-F_{n} \wedge F_{n+1}\right)+n\left(F_{n} \wedge F_{n-1}-I F_{n-1} \wedge I F_{n}\right)
$$

we get

$$
\begin{aligned}
T \xi= & \sum_{n=0}^{N} c_{n}(\lambda-n)\left(I F_{n+1} \wedge I F_{n}\right)+\sum_{n=-1}^{N-1} c_{n+1}(n+1)\left(I F_{n+1} \wedge I F_{n}\right) \\
& +\sum_{n=0}^{N} c_{n}(\lambda-n)\left(F_{n+1} \wedge F_{n}\right)+\sum_{n=-1}^{N-1} c_{n+1}(n+1)\left(F_{n+1} \wedge F_{n}\right) \\
= & \sum_{n=0}^{N-1}\left(c_{n}(\lambda-n)+c_{n+1}(n+1)\right)\left(\left(I F_{n+1} \wedge I F_{n}\right)+\left(F_{n+1} \wedge F_{n}\right)\right) \\
& +c_{N}(\lambda-N)\left(F_{N+1} \wedge F_{N}\right) .
\end{aligned}
$$

This term vanishes if and only if

$$
c_{n+1}=-\frac{\lambda-n}{n+1} c_{n} \text { for } 0 \leqq n \leqq N-1
$$

which means

$$
c_{n}=(-1)^{n}\left(\begin{array}{l}
\lambda \\
n
\end{array}\right) c_{0} \text { for } 0 \leqq n \leqq N
$$

From the equation $[U, T]=2 H$ we see that all the elements annihilated by $U$ and $T$ are 
also annihilated by $H$ and thus constitute already the null space of the $\operatorname{sl}(2, \mathbb{R})$-action. So we have proved that for $\lambda$ odd we have

$$
\left(\wedge^{2} V^{\lambda}\right)_{0}=\mathbb{R}\left(\sum_{n=0}^{N}(-1)^{n}\left(\begin{array}{l}
\lambda \\
n
\end{array}\right)\left(F_{n} \wedge I F_{n}\right)\right) .
$$

Propositions 1 and 2 now yield immediately:

Theorem 1. Let $V$ be an irreducible $\mathrm{sl}(2, \mathbb{R})$-module.

There is a non-zero invariant symplectic form on $V$ if and only if the dimension of $V$ is even. Then this invariant symplectic form is unique up to a scalar factor.

We just proved that an $\mathrm{sl}(2, \mathbb{R})$-module of even dimension possesses an invariant symplectic form which is unique up to a scalar. In the next proposition we determine this form explicitly.

Proposition 3. Let $V$ be an irreducible $\mathrm{sl}(2, \mathbb{R})$-module of even dimension with basis vectors $F_{n}, I F_{n}$ as above. Then an invariant symplectic form on $V$ is given by

$$
\beta(v, w)=\sum_{n=0}^{N} \frac{(-1)^{n}}{\left(\begin{array}{l}
\lambda \\
n
\end{array}\right)}\left(v_{n} w_{n}^{\prime}-v_{n}^{\prime} w_{n}\right)
$$

where $v=\sum_{n}\left(v_{n} F_{n}+v_{n}^{\prime} I F_{n}\right)$ and $w=\sum_{n}\left(w_{n} F_{n}+w_{n}^{\prime} I F_{n}\right)$. In other words: With respect to the basis $\left(F_{0}, I F_{0}, F_{1}, I F_{1}, \ldots, F_{n}, I F_{n}\right)$, the form $\beta$ is represented by the matrix

$$
\left(\begin{array}{llll}
A_{0} & & & \\
& A_{1} & & \\
& & \ddots & \\
& & A_{N}
\end{array}\right), \quad \text { where } A_{n}=\frac{(-1)^{n}}{\left(\begin{array}{ll}
\lambda \\
n
\end{array}\right)}\left(\begin{array}{rr}
0 & 1 \\
-1 & 0
\end{array}\right)
$$

Proof. Writing 


$$
\xi:=\sum_{n=0}^{N}(-1)^{n}\left(\begin{array}{l}
\lambda \\
n
\end{array}\right)\left(F_{n} \wedge I F_{n}\right)
$$

an invariant symplectic form $\hat{\beta}$ on $V^{*}$ is given by

$$
\begin{aligned}
\tilde{\beta}\left(\phi_{1}, \phi_{2}\right) & =(\Omega \xi)\left(\phi_{1}, \phi_{2}\right)=(\Omega \xi)\left(\phi_{1} \wedge \phi_{2}\right)=\left(\phi_{1} \wedge \phi_{2}\right)(\xi) \\
& =\sum_{n=0}^{N}(-1)^{n}\left(\begin{array}{l}
\lambda \\
n
\end{array}\right) \operatorname{det}\left(\begin{array}{ll}
\phi_{1}\left(F_{n}\right) & \phi_{1}\left(I F_{n}\right) \\
\phi_{2}\left(F_{n}\right) & \phi_{2}\left(I F_{n}\right)
\end{array}\right)
\end{aligned}
$$

If we define $f: V \rightarrow V^{*}$ by $\tilde{\beta}(\phi, f(v))=\phi(v)$ and put $\phi_{v}:=f(v)$, then the desired form $\beta$ on $V$ is given by $\beta(v, w):=\widetilde{\beta}\left(\phi_{v}, \phi_{w}\right)=\phi_{v}(w)$. Now if $v=\sum_{n}\left(v_{n} F_{n}+v_{n}^{\prime} I F_{n}\right)$ then

$$
\begin{aligned}
\phi\left(\sum_{n=0}^{N}\left(v_{n} F_{n}+v_{n}^{\prime} I F_{n}\right)\right) & =\phi(v)=\tilde{\beta}\left(\phi, \phi_{v}\right) \\
& =\sum_{n=0}^{N}(-1)^{n}\left(\begin{array}{l}
\lambda \\
n
\end{array}\right) \operatorname{det}\left(\begin{array}{ll}
\phi\left(F_{n}\right) & \phi\left(I F_{n}\right) \\
\phi_{v}\left(F_{n}\right) & \phi_{v}\left(I F_{n}\right)
\end{array}\right) \\
& =\phi\left(\sum_{n=0}^{N}(-1)^{n}\left(\begin{array}{l}
\lambda \\
n
\end{array}\right)\left(\phi_{v}\left(I F_{n}\right) F_{n}-\phi_{v}\left(F_{n}\right) I F_{n}\right)\right)
\end{aligned}
$$

Taking $\phi^{-1}$ on both sides and comparing coefficients yields

$$
-\phi_{v}\left(F_{n}\right)=\frac{v_{n}^{\prime}}{(-1)^{n}\left(\begin{array}{l}
\lambda \\
n
\end{array}\right)}, \quad \phi_{v}\left(I F_{n}\right)=\frac{v_{n}}{(-1)^{n}\left(\begin{array}{l}
\lambda \\
n
\end{array}\right)}
$$

3. The uniqueness of the Levi complement

Theorem 2. Let $L$ be a Lie algebra with cone potential and $H$ a compactly embedded Cartan algebra of $L$. Furthermore, let $S$ be any Levi complement adapted to $H$ such that $[H \cap R, S]=\{0\}$. Then for any root $\omega$ either $L^{\omega} \subseteq S$ or $L^{\omega} \subseteq R$.

We shall postpone the proof for a moment. Given this theorem, we can readily prove:

Theorem 3. Let $L$ be a Lie algebra with cone potential and $H$ a compactly embedded 
Cartan algebra of $L$. If $S$ and $S^{\prime}$ are Levi complements adapted to $H$ such that $[H \cap R, S]=\left[H \cap R, S^{\prime}\right]=\{0\}$, then $S=S^{\prime}$.

Proof. We set $\Sigma:=\bigoplus_{L^{\circ} \cap R=\{0\}} L^{\omega}$. By Theorem 2 above, we have

$$
S \cap L^{+}=S^{\prime} \cap L^{+}=\Sigma
$$

and $H+S=H+S^{\prime}=H+\Sigma$, which is a reductive algebra. But the semisimple part of a reductive algebra is uniquely determined, being just its commutator algebra. So $S=S^{\prime}=[H+\Sigma, H+\Sigma]$.

Proof of Theorem 2. We want to show that for $\omega \in \Omega$ either $L^{\omega} \subseteq R$ or $L^{\omega} \subseteq S$. Since $L^{\omega}=\left(L^{\omega} \cap R\right) \oplus\left(L^{\omega} \cap S\right)$, it is enough to show that we cannot have $L^{\omega} \cap S \neq\{0\}$ and $L^{\omega} \cap R \neq\{0\}$ at the same time. Let us assume $L^{\omega} \cap S \neq\{0\}$ and $L^{\omega} \cap R \neq\{0\}$ to obtain a contradiction. We pick an element $s \neq 0$ in $L^{\omega} \cap S$. Then

$$
\sigma:=\mathbb{R} I s+\mathbb{R} s+\mathbb{R}[I s, s] \subseteq\left(L^{\omega} \cap S\right) \oplus \mathbb{R}[I s, s]
$$

is isomorphic to $\operatorname{sl}(2, \mathbb{R})$ if $\omega \in \Omega_{P}$ and isomorphic to $\operatorname{so}(3)$ if $\omega \in \Omega_{K}$. (There is no other possibility, since the complexification of $\sigma$ must be isomorphic to $\operatorname{sl}(2, \mathbb{C})$ due to the structure theory of complex semisimple Lie algebras.) Now let us consider* the vector space

$$
V:=\sum_{k \operatorname{ker} v=\operatorname{ker} \omega}\left(L^{v} \cap R\right)
$$

This is a $\sigma$-module, because $\left[L^{\omega}, L^{v}\right] \subseteq L^{\omega+\nu}+L^{\omega-\nu}$. We claim that its zero module $V_{0}$ with respect to the action of $[I s, s]$ alone is trivial. Indeed, if $x=\sum_{v} x_{v} \in V_{0}$, then $0=[[I s, s], x]=\sum_{v} v([I s, s]) I x_{v}$, so if $x_{v} \neq 0$ then $[I s, s] \in \operatorname{ker} v=\operatorname{ker} \omega$ contradicting the fact that $\omega([I s, s]) \neq 0$. Furthermore, any irreducible $\sigma$-module of $V$ has even dimension because it decomposes into two-dimensional subspaces which are invariant under the action of $[I s, s] \in H$. Thus if $\sigma \cong \operatorname{sl}(2, \mathbb{R})$ then

$$
V=\bigoplus_{\lambda \text { odd }} n_{\lambda} V^{\lambda}
$$

where $V^{\lambda}$ is a submodule of type $\lambda$ as described in the second paragraph with multiplicity $n_{\lambda} \in \mathbb{N}_{0}$. Since $\omega([I s, s])>0$ for $\sigma \cong s l(2, \mathbb{R})$, we can assume without loss of generality that $\omega([I s, s])=2$ (otherwise replace $s$ by $s / \sqrt{\omega([I s, s])}$ ). so that $[I s, s]$ corresponds to the element $U \in \operatorname{si}(2, \mathbb{R})[6,(3.4)$, p. 62]. Thus the real weight $\omega$ takes the value 2 on $[I s, s]$. On the other hand, the minimum subspaces of $V^{\lambda}$ which are invariant under $[I s, s]$ are the planes $V_{n}^{\lambda}=\mathbb{R} F_{n} \oplus \mathbb{R} I F_{n}$, and the values of the real weights for the

*This idea is due to Karl-Hermann Neeb who made me aware of a mistake in the original manuscript. 
spaces $V_{n}^{\lambda}$ on $(I s, s]$ are the numbers $\lambda-2 n$ which are odd since $\lambda$ is odd. This is clearly a contradiction, and we have ruled out the possibility that $\sigma \cong \operatorname{sl}(2, \mathbb{R})$.

Therefore, $\sigma \cong \operatorname{so}(3)$. In this case $\omega([I s, s])<0$, and after a suitable normalization we can assume that $\omega([I s, s])=-2$. Then $[I s, s]$ corresponds to the element $2 e_{1} \in \operatorname{so}(3)[6$, (3.4), p. 62]. Now we have seen that $[I s, s]$ acts invertibly on $V$ and hence on all irreducible subspaces; hence no real weight takes the value -2 on $[I s, s]$ due to the remarks following Proposition 1. Thus we have also ruled out the possibility that $\sigma \cong \operatorname{so}(3)$ which is the desired contradiction.

In the introduction we observed a partition of the root system $\Omega$ into the classes $\Omega_{K}$ and $\Omega_{P}$. Now, as a consequence of Theorem 2, the set $\Omega$ also decomposes into the disjoint classes

$$
\Omega_{R}:=\left\{\omega \in \Omega \mid L^{\omega} \subseteq R\right\}=\left\{\omega \in \Omega \mid L^{\omega} \cap R \neq\{0\}\right\}
$$

and

$$
\Omega_{S}:=\left\{\omega \in \Omega \mid L^{\omega} \subseteq S\right\}=\left\{\omega \in \Omega \mid L^{\omega} \cap S \neq\{0\}\right\} .
$$

Now we characterize the elements of $H \cap R$ with the help of the $S$-roots.

Proposition 4. The following equivalence holds:

$$
h \in H \cap R \Leftrightarrow \omega(h)=0 \text { for all } \omega \in \Omega_{s} .
$$

Proof. " $\Rightarrow$ " Let $\omega \in \Omega_{s}$ so that $L^{\omega} \subseteq S$. Fix an element $s \neq 0$ in $L^{\omega}$. Then for all $h \in H \cap R$, we have $\omega(h) I s=[h, s] \in[H \cap R, S]=\{0\}$, whence $\omega(h)=0$.

" $\Leftarrow$ " Let $h=h_{R}+h_{s}$. For all $s=s_{0}+\sum_{\omega \in \Omega_{s}} s_{\omega} \in S$ we have

$$
0=\sum_{\omega \in \Omega_{S}} \omega(h) I s_{\omega}=[h, s]=\underbrace{\left[h_{R}, s\right]}_{\in R}+\underbrace{\left[h_{s}, s\right]}_{\in S}
$$

Thus $\left[h_{R}, s\right]=\left[h_{s}, s\right]=0$. As $s \in S$ was arbitrary, $h_{S} \in Z(S)=\{0\}$. Consequently, $h=$ $h_{R} \in R$.

Note that this is a perfect parallel to the characterization of the centre of $K_{H}$ given by

$$
h \in Z\left(K_{H}\right) \Leftrightarrow \omega(h)=0 \text { for all } \omega \in \Omega_{K} .
$$

\section{Propostion 5.}

(a) $\Omega_{K} \subseteq \Omega_{S}$.

(b) $H \cap R \subseteq Z\left(K_{H}\right)$.

(c) $\left(K_{H}\right)^{\prime}=\left[K_{H}, K_{H}\right] \subseteq S$.

(d) Putting $Z_{0}:=Z\left(K_{H}\right) \cap(H \cap S)$ and $H^{\prime}:=H \cap\left(K_{H}\right)^{\prime}$ we have

$$
H=\underbrace{(H \cap R) \oplus \overbrace{Z_{0}}^{H} \oplus H^{\prime} .}_{=Z\left(K_{H}\right)}
$$


Proof. (a) Suppose $L^{\omega} \subseteq R$ for some $\omega \in \Omega_{K}$. Fix an element $x \in L^{\omega} \backslash\{0\}$. Then $H+\mathbb{R} x+\mathbb{R} I x$ is a compactly embedded subalgebra of the solvable algebra $R$, hence abelian. But this contradicts the cone potential of $L$.

(b) This is an immediate consequence of part (a) and Proposition 4. Indeed, if $h \in H \cap R$, then $\omega(h)=0$ for all $\omega \in \Omega_{s} \supseteq \Omega_{K}$, whence $h \in Z\left(K_{H}\right)$.

(c) As a consequence of (a), we have

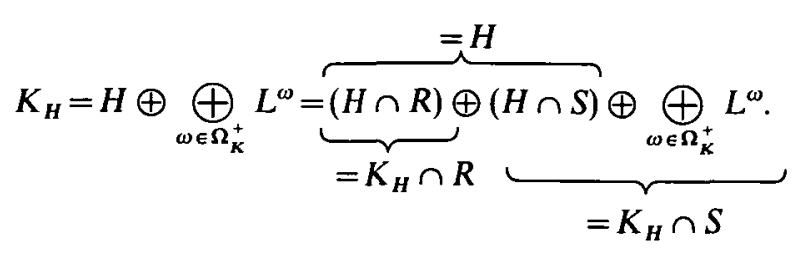

This implies $\left[K_{H}, K_{H}\right] \subseteq S$ because $[H \cap R, S]=\{0\}$.

(d) First of all, if $z \in Z\left(K_{H}\right)$ is decomposed as $z=h_{R}+h_{S}$, then $h_{S}=z-h_{R} \in Z\left(K_{H}\right)$ since $H \cap R \subseteq Z\left(K_{H}\right)$, whence $h_{S} \in Z_{0}$. Secondly, if $h \in H_{S}$ is decomposed as $h=z+h^{\prime}$, then $z=h-h^{\prime} \in H \cap S$ since $H^{\prime} \subseteq H \cap S$.

Note that $H_{S}:=H \cap S$ is a compactly embedded Cartan algebra of $S$; the maximal compactly embedded subalgebra of $S$ containing $H_{S}$ is

$$
K_{H_{s}}=\underbrace{Z_{0}}_{=Z\left(K_{H_{s}}\right)} \oplus \underbrace{\left(K_{H}\right)^{\prime}}_{=\left(K_{H_{s}}\right)^{\prime}} .
$$

Let us note at the end how the various sets of roots mentioned above behave under the Weyl group action. Here the Weyl group $\mathscr{W}$ of $(L, H)$ is the set of all equivalence classes $[\alpha]$ where $\alpha \in \overline{\left\langle e^{\text {ad } L}\right\rangle}$ with $\alpha(H)=H$ and $\alpha \sim \beta$ if and only if $\alpha \equiv \beta$ on $H$.

\section{Proposition 6.}

(a) The Weyl group $\mathscr{W}$ operates on $\Omega$ via

$$
[\alpha] * \omega:=\omega \circ \alpha^{-1}
$$

we have $L^{\omega \circ \alpha^{-1}}=\alpha\left(L^{\omega}\right)$.

(b) For all $[\alpha] \in \mathscr{W}$, the map $\left.\alpha\right|_{L^{+}}$preserves the complex structure $I$ of $L^{+}$; i.e., $\alpha \circ I=$ lo $\alpha$ on $\mathrm{L}^{+}$.

(c) The sets $\Omega_{K}, \Omega_{P}, \Omega_{R}$ and $\Omega_{S}$ are invariant under the Weyl group action.

Proof. (a) There is a root $\lambda$ of the pair $\left(L_{C}, H_{C}\right)$ such that $\omega=-\left.i \lambda\right|_{H}$; then $L^{\omega}=\left(L_{\mathbb{C}}^{\lambda} \oplus L_{\mathbb{C}}^{-\lambda}\right) \cap L$. Now let $[\alpha] \in \mathscr{W}$. We consider $\alpha$ as an automorphism of $L_{\mathbb{C}}$ leaving invariant the real form $L$. Then it suffices to show that $\lambda \circ \alpha^{-1}$ is a complex root satisfying $L_{\mathbb{C}}^{\lambda \circ \alpha^{-1}}=\alpha\left(L_{\mathbb{C}}^{\lambda}\right)$. But this is true, because for all $x \in L_{\mathbb{C}}^{\lambda}$ and all $h \in H_{\mathbb{C}}$ we have 


$$
[h, \alpha x]=\alpha[\underbrace{\alpha^{-1} h}_{\in H_{\mathbb{C}}}, \underbrace{x}_{\in L_{\mathbb{C}}^{\lambda}}]=\alpha\left(\lambda\left(\alpha^{-1} h\right) x\right)=\left(\lambda \circ \alpha^{-1}\right)(h) \alpha x .
$$

(b) Let $x \in L^{\omega}$ and choose $h \in H$ such that $\omega(h) \neq 0$. Now we have $[h, x]=\omega(h) l x$. Thus on the one hand, we have

$$
\alpha[h, x]=\alpha(\omega(h) I x)=\omega(h) \alpha I x .
$$

On the other hand, we have

$$
\alpha[h, \alpha x]=[\underbrace{\alpha h,}_{\in H \in L^{\omega \circ \alpha^{-1}}} \underbrace{\alpha x}]=\left(\omega \circ \alpha^{-1}\right)(\alpha h) I \alpha x=\omega(h) I \alpha x .
$$

A comparison yields $\alpha I x=I \alpha x$. Since $L^{+}=\bigoplus_{\omega \in \Omega^{+}} L^{\omega}$, we have $\alpha \circ I=I \circ \alpha$.

(c) We have $\alpha(R) \subseteq R$ for all $\alpha \in \overline{\left\langle e^{\text {ad } L}\right\rangle}$ because $R$ is an ideal of $L$. In particular, for all $[\alpha] \in \mathscr{W}$ the following equivalences hold:

$$
\omega \in \Omega_{R} \Leftrightarrow L^{\omega} \subseteq R \Leftrightarrow L^{\omega \circ a^{-1}}=\alpha\left(L^{\omega}\right) \subseteq R \Leftrightarrow \omega \circ \alpha^{-1} \in \Omega_{R}
$$

Thus the invariance of $\Omega_{R}$ (and hence also that of $\Omega_{S}$ ) is shown.

If $[\alpha] \in \mathscr{W}$, then $\alpha \in \overline{\left\langle e^{\text {ad } K_{H}}\right\rangle}$, whence $\alpha\left(K_{H}\right) \subseteq K_{H}$. As a consequence, the following equivalences hold:

$$
\omega \in \Omega_{K} \Leftrightarrow L^{\omega} \subseteq K_{H} \Leftrightarrow L^{\omega \circ \alpha^{-1}}=\alpha\left(L^{\omega}\right) \subseteq K_{H} \Leftrightarrow \omega \circ \alpha^{-1} \in \Omega_{K}
$$

Thus the invariance of $\Omega_{K}$ (hence also that $\Omega_{K}$ ) is shown.

\section{An example}

Let $N$ be the Heisenberg algebra, i.e.,

$$
N=\mathbb{R}^{2} \times \mathbb{R} \quad \text { with }\left[\left(\begin{array}{l}
u \\
t
\end{array}\right),\left(\begin{array}{l}
u^{\prime} \\
t^{\prime}
\end{array}\right)\right]=\left(\begin{array}{c}
0 \\
\operatorname{det}\left(u, u^{\prime}\right)
\end{array}\right) .
$$

We consider the semidirect product $L=N \rtimes_{\phi} \operatorname{sl}(2, \mathbb{R})$ where $\operatorname{sl}(2, \mathbb{R})$ acts on $N$ as an algebra of derivations via

$$
\phi(A)\left(\begin{array}{c}
u \\
t
\end{array}\right)=\left(\begin{array}{c}
A u \\
0
\end{array}\right)
$$

Explicitly, we have

$$
L=\mathbb{R}^{2} \times \mathbb{R} \times \operatorname{sl}(2, \mathbb{R}) \quad \text { with }\left[\left(\begin{array}{c}
u \\
t \\
A
\end{array}\right),\left(\begin{array}{c}
u^{\prime} \\
t^{\prime} \\
A^{\prime}
\end{array}\right)\right]=\left(\begin{array}{c}
A u^{\prime}-A^{\prime} u \\
\operatorname{det}\left(u, u^{\prime}\right) \\
A A^{\prime}-A^{\prime} A
\end{array}\right)
$$


Clearly, $R:=N$ is the radical of this algebra, and $S:=\operatorname{sl}(2, \mathbb{R})$ is a Levi complement. One readly verifies that

$$
\operatorname{comp} L=(\{0\} \times \mathbb{R} \times\{0\}) \cup\left(\mathbb{R}^{2} \times \mathbb{R} \times C\right)
$$

where $C=(\operatorname{compsl}(2, \mathbb{R})) \backslash\{0\}=\left\{\left(\begin{array}{cc}a & b \\ c & -a\end{array}\right) \mid a^{2}+b c<0\right\}$. Also,

$$
H:=\{0\} \times \mathbb{R} \times \mathbb{R} u \quad\left(U:=\left(\begin{array}{cc}
0 & 1 \\
-1 & 0
\end{array}\right)\right)
$$

is a compactly embedded Cartan algebra of $L$. The condition $[H \cap R, S]=\{0\}$ holds because $H \cap R=\{0\} \times \mathbb{R} \times\{0\}$ is the centre of $L$.

We define $\omega: H \rightarrow \mathbb{R}$ by $\omega(0, t, r U):=r$. Then it is easy to verify that $\Omega=\{ \pm \omega, \pm 2 \omega\}$. The root spaces are

$$
L^{\omega}=L^{-\omega}=\mathbb{R}^{2} \times\{0\} \times\{0\}, \quad L^{2 \omega}=L^{-2 \omega}=\{0\} \times\{0\} \times\left\{\left(\begin{array}{cc}
s & t \\
t & -s
\end{array}\right)\right\}
$$

the complex structure $I$ on $L^{+}=L^{\omega} \oplus L^{2 \omega}$ is given by

$$
I\left(\left(\begin{array}{l}
x \\
y
\end{array}\right), 0,\left(\begin{array}{cc}
s & t \\
t & -s
\end{array}\right)\right)=\left(\left(\begin{array}{c}
y \\
-x
\end{array}\right), 0,\left(\begin{array}{cc}
t & -s \\
-s & -t
\end{array}\right)\right)
$$

We see that $\Omega_{R}=\{ \pm \omega\}$ and $\Omega_{S}=\{ \pm 2 \omega\}$. Since $K_{H}=H$ in this example, we have $\Omega_{K}=\emptyset$ and $\Omega_{P}=\Omega$.

Let us remark that the algebra $L$ possesses two one-parameter families of invariant cones (where one family consists just of the negatives of the cones in the other family). For details, see [6, (10.4), pp. 143-144].

\section{REFERENCES}

1. Theodor Bröcker and Tammo tom Dieck, Representations of Compact Lie Groups (Springer, New York-Berlin-Heidelberg-Tokyo, 1985).

2. Nicolas Bourbaki, Groupes et algèbres die Lie, Chapitres 7 et 8 (Hermann, Paris, 1975).

3. Norbert Dörr, sl(2)-Tripel in reellen Lie-Algebren (Thesis, Darmstadt, 1988).

4. Joachim Hilgert, Karl Heinrich Hofmann and Jimmie D. Lawson, Lie Groups, Convex Cones, and Semigroups (Oxford University Press, Oxford, 1989).

5. Jean-Pierre Serre, Algèbres de Lie semi-simples complexes (Benjamin, New York-Amsterdam, 1966). 
6. Karlheinz Spindler, Invariante Kegel in Liealgebren, Mitt. Math Sem. Giessen 188 (1988), $1-159$.

7. Mitsuo Suglura, Unitary Representations and Harmonic Analysis (Kodansha, Tokyo, 1975).

Department of Mathematics

Louisiana State University

BATON ROUGE

LA 70803, USA 